\title{
Serum thyrotropin, thyroxine and free thyroxine concentrations as predictors of mortality in critically ill puppies with parvovirus infection: a model for human paediatric critical illness?
}

\author{
Johan P. Schoeman, Michael E. Herrtage \\ Department of Companion Animal Clinical Studies, Faculty of Veterinary Science, University \\ of Pretoria, Private bag X04, Onderstepoort, Pretoria 0110, South Africa \\ Department of Veterinary Medicine, University of Cambridge, Madingley Road, Cambridge \\ CB3 OES, England, United Kingdom
}

With 2 tables and 1 figure

\begin{abstract}
This prospective case-controlled study investigated the role of the pituitaryethyroidal axis in the prediction of mortality in dogs. Serum thyrotropin, thyroxine and free thyroxine were measured at admission and daily until death or discharge in dogs ill with parvoviral diarrhoea. Serum thyroxine and free thyroxine in ill dogs were significantly lower than in controls and also significantly lower in nonsurvivors than in survivors on days 1-4. Serum thyrotropin at admission in ill dogs was similar to controls, but was significantly higher in nonsurvivors than in survivors. Thyrotropin concentrations became significantly lower in nonsurvivors vs survivors by day 4 .
\end{abstract}

Keywords: Animal model; Dog; Parvovirus

\section{Introduction}

Canine parvovirus is a significant worldwide canine pathogen which emerged as a clinical problem in 1978 and produces a disease, predominantly in dogs $<6$ months of age, characterized by severe enteritis, vomiting, haemorrhagic diarrhoea, sepsis and shock [1]. Parvoviruses are small, non-enveloped, single-stranded DNA viruses that exhibit a tropism for actively replicating cell populations of the intestinal crypt epithelium, lymphoid and haemopoietic tissues [2]. Intestinal tract damage secondary to viral infection increases the risk of bacterial translocation, subsequent coliform septicaemia, development of a systemic inflammatory response, sepsis and death [3]. A large proportion of infected dogs has measurable serum concentrations of endotoxin, which is a potent mediator of the systemic inflammatory response through its stimulation of the release of pro-inflammatory cytokines such as tumour necrosis factor [3]. These and other cytokines such as interleukin- 1 (IL-1) and interleukin-6 (IL-6) have been shown to suppress the pituitaryethyroidal axis in human critical illness [4,5].

Critical illness in humans is characterized by multiple and complex alterations in the thyroid axis [6,7]. As the severity of illness progresses, there is a reduction not only of serum triiodothyronine (T3), but also thyroxine (T4) and thyrotropin (TSH) concentrations [8]. Low concentrations of T4 and TSH have been shown to indicate prolonged illness and a poor prognosis in human critical care $[9,10]$, especially in neonates undergoing open heart surgery [11] and in children with bacterial sepsis and septic shock [12]. The lowest serum T4 concentrations were associated with death probabilities in excess of $80 \%$ [13]. Canine non-thyroidal illness is a wellknown cause of reduced thyroid hormone concentrations and more severe illness has been shown to exhibit lower T4 and fT4 concentrations [14]. Canine parvoviral infection shows promise as an animal model, particularly for human paediatric critical illness, because significant associations between mortality and low serum thyroxine activity have previously been shown in puppies with this disease [15]. In addition, these puppies have a disease process culminating in sepsis and septic shock, which is similar to the studies in children in which low thyroid hormones indicated a poor prognosis $[1,12]$.

The aim of the study reported here was to assess the association of serial basal serum fT 4 and TSH concentrations, in addition to the previously described serum total T4 concentrations, with mortality in puppies with parvoviral diarrhoea. The temporal association 
between altered serum TSH concentrations and low serum thyroxine concentrations will give insight into the factors affecting thyroid hormone production in canine critical illness. It will also establish if additional host responses similar to those found in humans can be documented in parvoviral infection in dogs.

\section{Materials and methods}

\subsection{Animal model}

This prospective study was performed on 63 puppies with severe parvoviral diarrhoea, admitted consecutively to the high care isolation ward at the Onderstepoort Veterinary Academic Hospital, South Africa. Seventeen healthy puppies admitted to the same hospital for routine vaccination were used as control dogs. The study was reviewed and approved by the animal use and care committee of the University of Pretoria. All owners signed a written consent form allowing daily blood samples to be obtained from their dogs.

Patients with a diagnosis of parvoviral diarrhoea that fulfilled the following criteria were considered eligible for inclusion in the study: their disease condition necessitated admission to the high care ward; their body weights were $>2.5 \mathrm{~kg}$; the diagnosis of parvoviral diarrhoea was confirmed by the detection of parvoviral particles on faeces by use of electron microscopy.

\subsection{Study design}

Blood samples in the patient population were collected prior to treatment on admission (day 1) and daily thereafter between 8:00 a.m. and 11:00 a.m., until death or hospital discharge. Blood samples from control dogs were collected once in the consulting room after they had waited a similar period as the study dogs in the reception area of the same hospital. Blood was collected from the jugular vein by needle venipuncture in all dogs and placed into plastic tubes for serum collection. Blood samples were allowed to clot and tubes were centrifuged within $1 \mathrm{~h}$ of collection. The serum was harvested and stored at $-80^{\circ} \mathrm{C}$ until analyzed.

\subsection{Assays}

All samples from the patient population were assayed in a single batch; samples from control dogs were assayed in a second batch. Serum T4, fT4 and TSH concentrations were determined in duplicate on a gamma counter with previously validated radioimmunoassay and radioimmunometric kits.

\subsection{Data analysis}

Within group serial daily differences were measured by use of the Friedman test for related samples. Differences in median serum T4, fT4 and TSH concentrations between nonsurvivor and survivor groups were analyzed by the Manne-Whitney U-test for nonparametric data, unless stated otherwise. Comparisons between nonsurvivors and survivors were only made up to day 4, after which only 3 dogs remained in the nonsurvivor group, precluding any further reliable comparisons between the outcome groups. For all comparisons and correlations, values of $P<0.05$ were considered significant. Values are reported as median and range or interquartile range (IQR). Statistical analysis was performed on a personal computer by the use of a commercial software package.

\section{Results}

\subsection{Patient population}

Signalment, historical and clinical data for the 3 groups of dogs are depicted in Table 1. Sixtythree dogs with parvoviral diarrhoea were included in the study. Thirteen dogs were in the nonsurvivor group and 50 in the survivor group. Of nonsurvivor group dogs, 4 died on admission (day 1), another dog died prior to sampling on day 2. Three dogs died during day 
3,2 dogs on day 4,1 on day 5 and the 2 remaining dogs on day 6 . Of the 13 dogs that died, 8 were male and 5 were female. Overall, 32 of $63(51 \%)$ dogs with parvoviral diarrhoea were male and $31(49 \%)$ were female. Nonsurvivor group dogs

were significantly younger, weighed significantly less and had a significantly shorter hospital stay than survivor group dogs. Duration of illness, time since last meal, body temperature, pulse rate, respiratory rate and haematocrit did not differ between the parvoviral diarrhoea groups (Table 1).

\subsection{Control population}

Healthy dogs consisted of 10 sexually intact males and 7 sexually intact females. Median age was 3 months (range, 2-13 months). Median body weight was $7 \mathrm{~kg}$ with a range of $2.5-24 \mathrm{~kg}$ (Table 1).

\subsection{Serum thyroxine and free thyroxine concentrations on admission}

Serum T4, fT4 and TSH data from day 1 to day 4 are depicted in Table 2. Median serum thyroxine and free thyroxine concentrations on admission (day 1 ) in all dogs with parvoviral diarrhoea were significantly $(P<0.001)$ lower than in control dogs. Median serum thyroxine and free thyroxine concentrations on admission of dogs with parvoviral diarrhoea were significantly $\left(P^{1 / 4} 0.016\right.$ and $P 1 / 40.009$, respectively) lower in nonsurvivors than in survivors (Fig. 1).

\subsection{Serum TSH concentrations on admission}

Median serum TSH concentrations on admission in all dogs with parvoviral diarrhoea were not significantly $\left(P^{1 / 4} 0.47\right)$ different from control dogs $(0.03 \mathrm{ng} / \mathrm{dl}[0.03-0.06 \mathrm{ng} / \mathrm{dl}] \mathrm{vs} 0.04$ $\mathrm{ng} / \mathrm{dl}$ [0.03-0.07 ng/dl], respectively). Median serum TSH concentrations on admission of dogs with parvoviral diarrhoea were significantly $\left(P^{1 / 4} 0.033\right)$ higher in nonsurvivors than in survivors

\subsection{Subsequent daily serum thyroxine and free thyroxine concentrations}

On day 2, the remaining 8 nonsurvivor group dogs had significantly $(P<0.05$ for both) lower median serum thyroxine and free thyroxine concentrations than the 47 survivor group dogs. On day 3 , the remaining 5 nonsurvivors had significantly $(P<0.05$ for both) lower median serum thyroxine and free thyroxine concentrations than the 38 survivors. On day 4 , the remaining 3 nonsurvivors had significantly $(P<0.01$ and $P<0.05$, respectively) lower median serum thyroxine and free thyroxine concentrations than the 26 survivors. The percentage of puppies in the nonsurvivor group with serum thyroxine and free thyroxine below the normal reference range increased from $87.5 \%$ on day 2 to $100 \%$ on day 4 . The Friedman test demonstrated that serum thyroxine and free thyroxine concentrations in survivor group dogs increased significantly $(P<0.01)$ from days 1 to 3 , whereas the nonsurvivor group did not show a significant change in either serum thyroxine or free thyroxine concentrations from days 1 to 3 (Table 2).

\subsection{Subsequent daily serum TSH concentrations}

Median serum TSH concentrations did not differ significantly between the survivor and nonsurvivor groups on days 2-4, but a much larger percentage of nonsurvivor group dogs had serum TSH concentrations below the limit of detection of the assay on days 3 and $4 ; 82 \%$ vs $68 \%$ and $100 \%$ vs $62 \%$ (Table 2 ).

\section{Discussion}

Median basal serum thyroxine and free thyroxine concentrations were lower in nonsurvivor group dogs than survivor group dogs on days 1-4, whereas the median concentrations in survivor group dogs increased over the same time period. This finding is consistent with the responses in the human pituitary-thyroidal axis noted in children with sepsis [12]. Basal serum thyroxine and free thyroxine concentrations were already lower in nonsurvivor group dogs 
than in survivor group dogs on day 1 , while the respective TSH concentrations of the nonsurvivor group were higher than the survivor group.

This seems to indicate that the thyroid axis, at least initially, is suppressed by factors other than the decreased production of TSH from the pituitary gland. It is also possible that the elevated TSH concentrations in the sicker dogs were due to a relatively diminished negative feedback on the pituitary gland by the lower thyroid hormone concentrations in this nonsurvivor group of dogs. According to the results of our study, serum thyroxine and free thyroxine concentrations are more sensitive predictors of mortality than $\mathrm{TSH}$, although we advise caution in over interpreting the marginal TSH differences. It is likely that the inability of this first generation canine TSH to read concentrations below $0.03 \mathrm{ng} / \mathrm{dl}$ is a major limitation in demonstrating differences in hormone concentrations between groups of sick dogs, in which all have values clustering below the current limit of detection. The development of second and third generation assays, such as those currently used for humans, should remove this difficulty in outcome prediction in canine critical illness.

The finding of elevated TSH concentrations during the initial stages of critical illness is entirely compatible with studies in human critical care [16]. It is known in humans that cytokines such as interleukin-6 exerts an inhibitory effect on the hypothalamic-pituitary-thyroid axis $[4,5]$. Results of our study, when seen in combination with a recently performed pilot study showing a positive correlation of IL- 6 with mortality in dogs admitted with systemic inflammatory response syndrome, may lend credence to this cytokine as being instrumental in the reduction in thyroid hormone concentrations seen in our dogs [17].

There appears to be a close association between the degree of serum thyroxine, free thyroxine and TSH perturbations and mortality in dogs with parvovirus diarrhoea. The results of our study, although agreeing with most studies in human $[9,12]$ and canine acute critical illness [15,18], are at odds with a study conducted on more chronically ill dogs admitted to an intensive care unit with disparate illnesses [19]. The neuroendocrine paradigms in acute vs chronic critical illness (at least in humans) have been shown to be very different [16] and indications are that the same may be true for dogs.

This study confirms that the hypothyroxinaemia observed in critical canine illness is initially not wholly of pituitary origin, but that decreased TSH from the pituitary is a later feature of the hormone profiles in puppies with parvoviral diarrhoea, as has been demonstrated in human critical illness [16]. The low TSH is a logical proximate cause of the low thyroid hormone concentrations as critical illness progresses, with probable low TRH in turn being responsible for the low TSH concentrations. Hypothalamic dysfunction has been clearly demonstrated in human critical illness, as evidenced by the reduction in other hypothalamic hormones such as FSH and LH in relation to disease severity [20]. These changes noted in the pituitary-thyroidal axis are quite possibly the effect on the hypothalamus of neural integration of multiple factors such as stress, starvation, glucocorticoids and cytokines, all of which has been demonstrated in puppies with parvovirus infection $[3,15]$.

\section{Acknowledgements}

This study was funded by the Clinical Studies Trust Fund of the European College of Veterinary Internal Medicine. The authors gratefully acknowledge the invaluable laboratory assistance of Mrs Lea Goddard, Elsbe Myburgh and Annemarie Human.

\section{References}

[1] R.V. Pollock, M.J. Coyne, Canine parvovirus, Vet. Clin. North Am. Small Anim. Pract. 23 (1993) 555-568.

[2] P.C.Meunier, B.J.Cooper,M.J.Appel,D.O.Slauson,Pathogenesisofcanine parvovirusenteritis:theimportanceofviremia,Vet.Pathol.22(1985)60-71.

[3] C.M. Otto, K.J. Drobatz, C. Soter, Endotoxemia and tumor necrosis factor activity in dogs with naturally occurring parvoviral enteritis, J. Vet. Intern. Med. 11 (1997) 65-70.

[4] H. Karga, P. Papaioannou, K.Venetsanou, F. Papandroulaki, L. Karaloizos, G. Papaioannou, P. Papapetrou, The role of cytokines and cortisol in the non-thyroidal illness syndrome following acute myocardial infarction, Eur. J. Endocrinol. 142 (2000) 236-242 [5] H. Monig, T. Arendt, M. Meyer, S. Kloehn, B. Bewig, Activation of the hypothalamopituitaryeadrenal axis in response to septic or non-septic diseases e implications for the euthyroid sick syndrome, Intensive Care Med. 25 (1999) 1402-1406. 
[6] E.M. Kaptein, D.A. Grieb, C.A. Spencer, W.S. Wheeler, J.T. Nicoloff, Thyroxine metabolism in the low thyroxine state of critical nonthyroidal illnesses, J. Clin. Endocrinol. Metab. 53 (1981) 764-771.

[7] I.J. Chopra, J.M. Hershman, W.M. Pardridge, J.T. Nicoloff, Thyroid function in nonthyroidal illnesses, Ann. Intern. Med. 98 (1983) 946-957.

[8] L.J. De Groot, Non-thyroidal illness syndrome is a manifestation of hypothalamic-pituitary dysfunction, and in view of current evidence, should be treated with appropriate replacement therapies, Crit. Care Clin. 22 (2006) 57-86.

[9] P.M. Rothwell, Z.F. Udwadia, P.G. Lawler, Thyrotropin concentration predicts outcome in critical illness, Anaesthesia. 48 (1993) 373-376.

[10] D.C. Ray, A. Macduff, G.B. Drummond, E. WilkinsonB.Adams, G.J. Beckett, Endocrine measurements in survivors and non-survivors from critical illness, Intensive Care Med. 28 (2002) 1301-1308.

[11] O. Dagan, B. Vidne, Z. Josefsberg, M. Phillip, D. Strich, E. Erez, Relationship between changes in thyroid hormone level and severity of the postoperative course in neonates undergoing open-heart surgery, Paediatr. Anaesth. 16 (2006) 538-542.

[12] D. Yildizdas, N. Onenli-Mungan, H. Yapicioglu, A.K. Topaloglu, Y. Sertdemir, B. Yuksel, Thyroid hormone levels and their relationship to survival in children with bacterial sepsis and septic shock, J. Pediatr. Endocrinol. Metab. 17 (2004) 1435e1442.

[13] L.S. Maldonado, G.H. Murata, J.M. Hershman, G.D. Braunstein, Do thyroid function tests independently predict survival in the critically ill? Thyroid 2 (1992) 119-123.

[14] L.B. Kantrowitz, M.E. Peterson, C. Melian, R. Nichols, Serum total thyroxine, total triiodothyronine, free thyroxine and thyrotropin concentrations in dogs with nonthyroidal disease, J. Am. Vet. Med. Assoc. 219 (2001) 765-769.

[15] J.P. Schoeman, A. Goddard, M.E. Herrtage, Serum cortisol and thyroxine concentrations as predictors of mortality in critically ill puppies with parvoviral diarrhea, J. Am. Vet. Med. Assoc. 231 (2007) 1534-1539.

[16] R.P. Peeters, Y. Debaveye, E. Fliers, T.J. Visser, Changes within the thyroid axis during critical illness, Crit. Care Clin. 22 (2006) 41-55.

[17] S. Rau, B. Kohn, C. Richter, N. Fenske, H. Kuchenhoff, K. Hartmann, T. Goebel, D. Kaspers, J. Hirschberger, Plasma IL-6 Response is Predictive for Severity and Mortality in Canine SIRS and Sepsis, in: Proceedings of the 16th Congress of the European College of Veterinary Internal medicine (2006), p. 36 Amsterdam, Netherland.

[18] J.P. Schoeman, P. Rees, M.E. Herrtage, Endocrine predictors of mortality in canine babesiosis caused by Babesia canis rossi, Vet. Parasitol. 148 (2007) 75-82.

[19] D.A. Elliott, L.G. King, C.A. Zerbe, Thyroid hormone concentrations in critically ill canine intensive care patients, J. Vet. Emerg. Crit. Care 5 (1995) 17-23.

[20] D.I. Spratt, P. Cox, J. Orav, J. Moloney, T. Bigos, Reproductive axis suppression in acute illness is related to disease severity, J. Clin. Endocrinol. Metab. 76 (1993) 1548-1554.

Table 1

Summary of the signalment, historical and clinical data in the control group and the survivor and nonsurvivor groups of dogs with parvoviral diarrhoea

\begin{tabular}{|c|c|c|c|}
\hline Parameter & $\begin{array}{l}\text { Control group (n } 1 / 417 \text { ), } \\
\text { median (range) }\end{array}$ & $\begin{array}{l}\text { Survivors ( } \mathrm{n} 1 / 450) \text {, } \\
\text { median (range) }\end{array}$ & 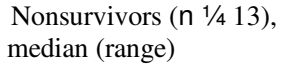 \\
\hline Age & 3 months $(2-13)$ & 4 months (1-9) & 3 months $(2-5)^{\mathrm{a}}$ \\
\hline Sex: M/F & $10 / 7$ & $24 / 26$ & $8 / 5$ \\
\hline Body weight & $7 \mathrm{~kg}(2.5-24)$ & $5.78 \mathrm{~kg}(2.5-44)$ & $4 \mathrm{~kg}(2.5-6.3)^{\mathrm{b}}$ \\
\hline Duration of illness & - & 4 days (1-9) & 3 days $(2-5)$ \\
\hline Time since last meal & $4 \mathrm{~h}(3.5-12)^{\mathrm{c}}$ & 48 h (12-96) & 32 h $(24-96)$ \\
\hline Length of hospital stay & $y-$ & 4 days (1-9) & 2 days $(0-5)^{\mathrm{c}}$ \\
\hline Body temperature & $38.8^{\circ} \mathrm{C}(38.3-39.8)$ & $38.7^{\circ} \mathrm{C}(34.8-40.2)$ & $38.5^{\circ} \mathrm{C}(37.1-39.5)$ \\
\hline Pulse rate & 152 bpm (100-188) & 141 bpm $(88-250)$ & 160 bpm (120-210) \\
\hline Respiratory rate & 30 bpm (20-96) & 34 bpm (16-120) & 40 bpm (20-112) \\
\hline Haematocrit & $0.361 / 1(0.23-0.50)$ & $0.381 / 1(0.08-0.61)$ & $0.361 / 1(0.22-0.60)$ \\
\hline
\end{tabular}

bpm $1 / 4$ Beats/breaths per minute.

a Significantly different at $P<0.05$.

${ }^{b}$ Significantly different at $P<0.01$.

${ }^{\mathrm{c}}$ Significantly different at $P<0.001$. 


\section{Table 2}

Results of the serum TT4, fT4 and TSH data in the control group and the survivor and nonsurvivor groups of dogs with parvoviral diarrhoea

\begin{tabular}{|c|c|c|c|c|}
\hline Analyte & $\begin{array}{l}\text { Admission (day } 1) \\
\text { parvo dogs }(n 1 / 463), \\
\text { control dogs }(n 1 / 417)\end{array}$ & $\begin{array}{l}\text { Day 2, parvo dogs } \\
(n-1 / 455)\end{array}$ & $\begin{array}{l}\text { Day 3, parvo dogs } \\
(n=1 / 443)\end{array}$ & $\begin{array}{l}\text { Day 4, parvo dogs } \\
(n 1 / 429)\end{array}$ \\
\hline \multicolumn{5}{|c|}{ TT4: median (IQR) } \\
\hline Survivors & $5.94 \mathrm{nmol} / \mathrm{l}(2.7-14.3)$ & $13.8 \mathrm{nmol} / 1(6.5-23)$ & $12.3 \mathrm{nmol} / 1(5.9-22.6)$ & $9.1 \mathrm{nmol} / 1(5.4-20.5)$ \\
\hline Nonsurvivors & $2.7 \mathrm{nmol} / 1(2.7-5.25)^{\mathrm{a}}$ & $2.7 \mathrm{nmol} / \mathrm{l}(2.7-7)^{\mathrm{a}}$ & $3.1 \mathrm{nmol} / 1(2.7-10)^{\mathrm{a}}$ & $2.7 \mathrm{nmol} / \mathrm{l}(2.7-2.7)^{\mathrm{b}}$ \\
\hline \multicolumn{5}{|l|}{ TT4: $n(\%)$} \\
\hline Control group & $0 / 17(0 \%)$ & & & \\
\hline Survivors & $38 / 48(79 \%)$ & $26 / 47(55 \%)$ & $24 / 38(63 \%)$ & $17 / 26(65 \%)$ \\
\hline Nonsurvivors & $12 / 13(92 \%)$ & $7 / 8(87.5 \%)$ & $5 / 5(100 \%)$ & $3 / 3(100 \%)$ \\
\hline \multicolumn{5}{|l|}{ fT4: median (IQR) } \\
\hline \multicolumn{5}{|l|}{ fT4: $n(\%)$} \\
\hline \multicolumn{5}{|l|}{$<1.3 \mathrm{pmol} / 1$} \\
\hline Control group & $0 / 17(0 \%)$ & & & \\
\hline Survivors & $28 / 47(60 \%)$ & $14 / 47(30 \%)$ & $15 / 38(39 \%)$ & $12 / 26(46 \%)$ \\
\hline Nonsurvivors & $11 / 13(85 \%)$ & $7 / 8(87.5 \%)$ & $4 / 5(80 \%)$ & $3 / 3(100 \%)$ \\
\hline \multicolumn{5}{|c|}{ TSH: median (IQR) } \\
\hline Control group & $0.04 \mathrm{ng} / \mathrm{dl}(0.03-0.07)$ & & & \\
\hline Survivors & $0.03 \mathrm{ng} / \mathrm{dl}(0.03-0.04)$ & $0.03 \mathrm{ng} / \mathrm{dl}(0.03-0.07)$ & $0.03 \mathrm{ng} / \mathrm{dl}(0.03-0.06)$ & $0.03 \mathrm{ng} / \mathrm{dl}(0.03-0.05)$ \\
\hline Nonsurvivors & $0.06 \mathrm{ng} / \mathrm{dl}(0.03-0.18)^{\mathrm{a}}$ & $0.03 \mathrm{ng} / \mathrm{dl}(0.03-0.33)$ & $0.03 \mathrm{ng} / \mathrm{dl}(0.03-0.05)$ & $0.03 \mathrm{ng} / \mathrm{dl}(0.03-0.03)$ \\
\hline \multicolumn{5}{|l|}{ TSH: $n(\%)$} \\
\hline
\end{tabular}

IQR $1 / 4$ interquartile range.

${ }^{a}$ Significantly different at $P<0.05$.

${ }^{b}$ Significantly different at $P<0.01$.

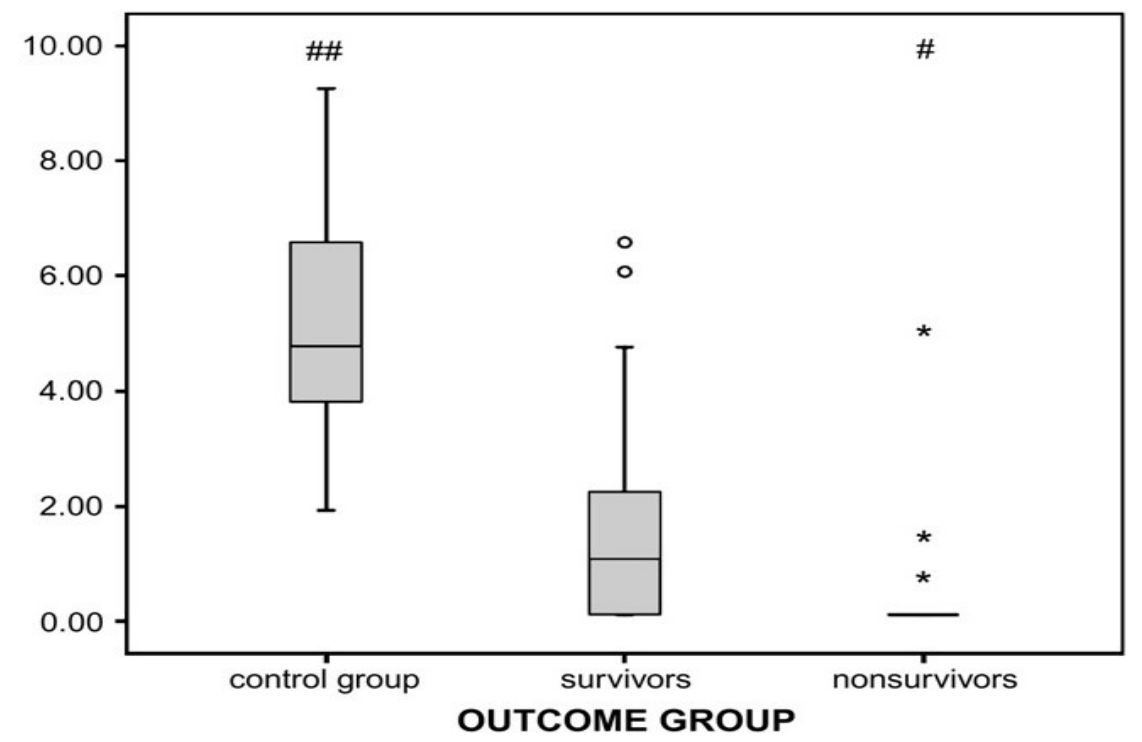


Fig. 1. Box plots of the admission serum-free thyroxine (fT4) concentrations in 17 control puppies and 63 puppies with parvoviral diarrhoea consisting of a survivor group $\left(n^{1 / 4} 50\right)$ and a nonsurvivor group $\left(n \frac{1 / 4}{13}\right)$. For each box plot, $T$ bars represent the main body of data, which is equal to the range in most instances (circles and asterisks indicate outliers). The box represents the IQR and the horizontal bar within the box is the median. \#\#, Control group significantly $(P<0.001)$ higher than both other groups. \#, Nonsurvivor group significantly $(P<0.05)$ lower than survivor group dogs. 